\title{
Minimum-Effort Waypoint-Following Guidance
}

\author{
Shaoming $\mathrm{He}^{*}$ \\ Cranfield University, Cranfield MK43 OAL, United Kingdom \\ Chang-Hun Lee ${ }^{\dagger}$ \\ Korea Advanced Institute of Science and Technology (KAIST), Daejeon, 34141, Republic of Korea \\ Hyo-Sang Shin ${ }^{\ddagger}$ \\ Cranfield University, Cranfield MK43 OAL, United Kingdom \\ Antonios Tsourdos $\S$ \\ Cranfield University, Cranfield MK43 OAL, United Kingdom
}

This paper addresses the problem of minimum-effort waypoint-following guidance with/without arrival angle constraints of an Unmanned Aerial Vehicle. By utilizing a linearized kinematics model, the proposed guidance laws are derived as the solutions of a linear quadratic optimal control problem with an arbitrary number of terminal boundary constraints. Theoretical analysis reveals that both optimal proportional navigation guidance and trajectory shaping guidance are special cases of the proposed guidance laws. The key feature of the proposed algorithms lies in their generic property. For this reason, the guidance laws developed can be applied to general waypoint-following missions with an arbitrary number of waypoints and an arbitrary number of arrival angle constraints. Nonlinear numerical simulations clearly demonstrate the effectiveness of the proposed formulations.

\section{Introduction}

Autonomous and reliable path following is a fundamental and key technology of Unmanned Aerial Vehicles (UAVs) to accomplish their mission objectives [1-4]. The primary objective of a path following guidance algorithm is to drive the vehicle to converge to a desired trajectory and remain on it thereafter. The desired path usually can be categorized into two classes: a continuous curve and a finite number of waypoints. Compared with continuous curve following, the generation of waypoints only requires the selection of several characteristic points and therefore is more computational efficient [5]. Besides, this approach does not require the concept of a look-ahead-distance [6, 7] or a virtual target on a continuous curve [8]. For these reasons, this paper aims to handle the issue of waypoint-following guidance.

\footnotetext{
*PhD Candidate, School of Aerospace, Transport and Manufacturing, College Road, AIAA Student Member, Email: shaoming.he.cn@gmail.com

$\dagger$ Assistant Professor, Department of Aerospace Engineering, Email: chleed fdcl.kaist.ac.kr (Corresponding Author)

${ }^{\ddagger}$ Reader, School of Aerospace, Transport and Manufacturing, College Road, Member AIAA, Email: h.shin@cranfield.ac.uk

§Professor, School of Aerospace, Transport and Manufacturing, College Road, Senior Member AIAA, Email: a.tsourdos@cranfield.ac.uk
} 
The problem of following a path has received massive interest in the robotics literature. In [9], the authors utilized vector fields to represent the desired headings to guide the UAV to follow a pre-designed path. The key feature of this approach is that both the heading error and the lateral distance error converge to zero asymptotically. By formulating the path following as a finite horizon regulation problem, model predictive control and receding-horizon control were also applied in [10-12] to minimize the distance tracking error. A nonlinear path following guidance law, adapted from the concept of pure pursuit, was suggested in [6] and its performance as well as stability were analyzed in [7]. Inspired by the development of missile guidance, the authors in $[8]$ applied pursuit guidance to follow a virtual target. The trajectory of the virtual target is specifically designed to cater for the requirement of path following. This concept was further modified by using trajectory shaping guidance (TSG) in [13, 14]. The advantage of utilization of TSG, compared with pure pursuit, lies in that TSG generates the same instantaneous radius of curvature as a curved path, thus providing accurate path following. In order to fully exploits the synergism between the guidance loop and the control loop, an integrated guidance-autopilot algorithm was proposed in [15] for UAV path following. By leveraging the properties of differential geometry of space curves, a three-dimensional nonlinear guidance law for UAV path following was developed in [16]. The almost-global asymptotic stability for a constant-curvature trajectory was also rigorously proved in this reference. Considering the turning rate limit, an algorithm was proposed in [17, 18] to find the globally shortest path through a given sequence of waypoints.

In [19], a linear quadratic optimal waypoint-following guidance was proposed to follow a straight line between two waypoints. The line segment changing point was also analytically derived by minimizing the magnitude of the required acceleration. Although this guidance law is optimal in each line segment, it fails to provide the global optimality when considers all waypoints. In practical flight, the energy consumption of a UAV governs the endurance of the vehicle. For this reason, numerical optimization approaches were utilized in [20, 21] to minimize energy consumption in waypoint following guidance. The drawback of using numerical algorithms is their relatively high computational complexity, which precludes their applications in low-cost small-scale UAVs. To alleviate this issue, the authors in [22] leveraged optimal impact angle guidance between every two consecutive waypoints to develop a globally energy-optimal guidance law for waypoint following of anti-ship missiles. However, the implementation of this algorithm requires offline parameter optimization to find the boundary conditions, e.g., passing angle and passing acceleration.

Intuitively, waypoint-following guidance can be viewed as a point-to-point guidance between two consecutive waypoints. This means that the well-developed missile guidance laws during last few decades can also be applied for UAV waypoint-following guidance. It is well-known that classical proportional navigation guidance (PNG) with a constant navigation gain three is energy-optimal for point-to-point guidance [23-25]. If a specific arrival angle or approach angle is required, TSG becomes the energy-optimal point-to-point guidance law [26-28]. However, it is unclear whether or not the total energy consumption is optimal for the case of multiple waypoints if we simply apply these two optimal guidance laws between two consecutive points. The difficulty of finding closed-form minimum-effort 


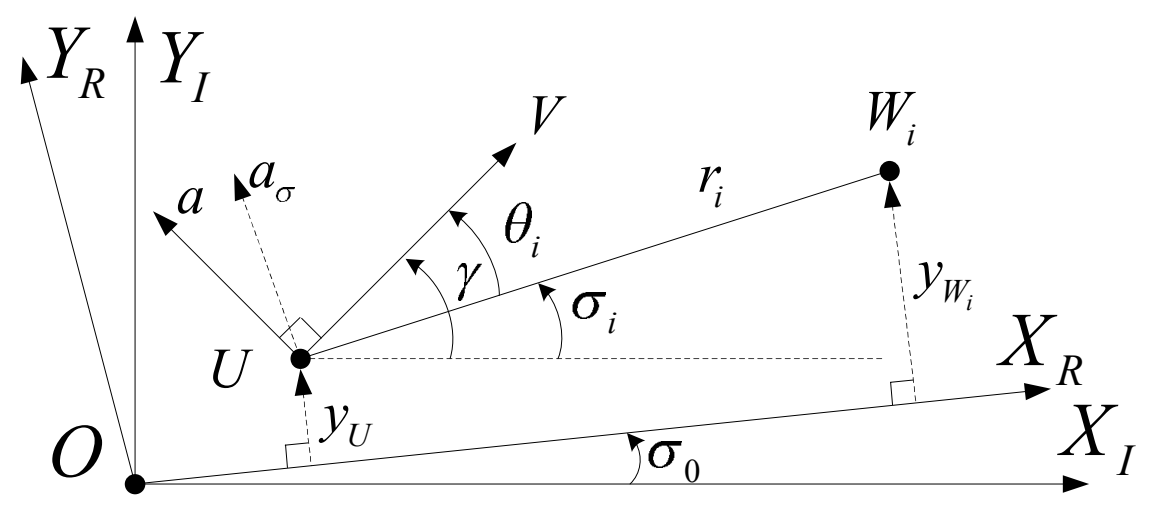

Fig. 1 Planar engagement geometry.

guidance law naturally arises in the multiple boundary conditions, e.g., multiple waypoint constraints and multiple angle constraints.

Motivated by the above observations, this paper aims to propose minimum-effort or energy-optimal guidance laws for UAV waypoint following. By formulating a finite-time linear regulation problem, two waypoint-following guidance laws are analytically derived using optimal control theory based on a linear kinematics model. The first guidance law is suited for the optimal waypoint following mission while the second one additionally considers partial arrival angle constraints, e.g., the UAV is required to pass several waypoints with specific flight path angles if it utilizes the terrain reference navigation as its navigation system. Theoretical analysis reveals that the proposed guidance laws reduce to classical PNG and TSG when there exists only one waypoint to be visited. The significant contribution of the proposed algorithm lies in its generic feature. That is, the proposed guidance law can be applied for energy-optimal waypoint following with arbitrary number of waypoints and arbitrary number of angle constraints. Nonlinear numerical simulations demonstrate that the proposed guidance laws help to increase the endurance of the vehicle, compared to waypoint-following guidance laws based on classical PNG and TSG.

The remainder of this paper is organized as follows. The backgrounds and preliminaries of this paper are stated in Sec. [I] Section III presents the details of the proposed guidance law for waypoint following, followed by the guidance law derived for optimal waypoint following with partial arrival angle constraint shown in Sec. IV] Finally, some simulation results and conclusions are offered.

\section{Backgrounds and Preliminaries}

This paper assumes that the UAV is equipped with a high-performance low-level flight control system that provides roll, pitch and yaw stability of the UAV as well as velocity tracking, heading and altitude hold functions. This study aims to design guidance inputs to this low-level controller for energy-optimal waypoint following in a two-dimensional geometry. 


\section{A. Nonlinear Kinematics}

Consider there exist $N$ waypoints that will be visited by the UAV. The relative geometry between the UAV and the $i$ th waypoint is shown in Fig. 1, where the symbols $U$ and $W_{i}$ denote the UAV and the $i$ th waypoint, respectively. The frame $X_{I} O Y_{I}$ is a inertial coordinate. The notation $\gamma$ stands for the UAV's flight path angle. In general, the UAV speed is pre-determined according to specific mission objective and is maintained by an engine controller. For this reason, we assume that the UAV is flying with a constant velocity $V$. The UAV changes its direction through the lateral acceleration $a$. The variable $a_{\sigma}$ denotes the UAV acceleration normal to the line-of-sight (LOS) direction. For simplicity, the UAV is assumed to be an ideal point mass model, i.e., the autopilot has no time delay. The variables $r_{i}$ and $\sigma_{i}$ represent the relative range and LOS angle between the UAV and the $i$ th waypoint. Based on the principles of kinematics, the differential equations describing the engagement geometry, depicted in Fig. 1. are formulated as

$$
\begin{aligned}
\dot{r_{i}} & =-V \cos \theta_{i} \\
\dot{\sigma_{i}} & =-\frac{V \sin \theta_{i}}{r_{i}} \\
\dot{\gamma} & =\frac{a}{V}, \quad i \in\{1,2, \cdots, N\}
\end{aligned}
$$

where $\theta_{i}$ denotes the velocity lead angle and can be determined as

$$
\theta_{i}=\gamma-\sigma_{i}
$$

Without loss of generality, assume that the $N$ waypoints are ordered by the increase of their corresponding passing times $t_{f, i}$ as $t_{f, i}<t_{f, i+1}$. Around the ideal approaching course, the waypoint passing time can be approximated by

$$
t_{f, i}=\frac{r_{i}(0)}{V}, \quad i \in\{1,2, \cdots, N\}
$$

where $r_{i}(0)$ denotes the initial relative range between the UAV and the $i$ th waypoint.

\section{B. Linearized Kinematics}

In this paper, optimal guidance laws will be derived based on a linearized model around the desired approaching course. For the purpose of kinematics linearization, a new frame called the reference frame $X_{R} O Y_{R}$ is introduced, as depicted in Fig. 1 This frame is rotated from the inertial frame by $\sigma_{0}$, which is the reference angle. Let $y_{i}=y_{W_{i}}-y_{U}$ be the relative displacement between the UAV and the $i$ th waypoint normal to the $X_{R}$ axis. In the reference frame, the 
engagement kinematics can be expressed as

$$
\begin{aligned}
& \dot{y}_{i}=v_{i} \\
& \dot{v}_{i}=-a_{\sigma} \cos \left(\sigma_{i}-\sigma_{0}\right), \quad i \in\{1,2, \cdots, N\}
\end{aligned}
$$

where $v_{i}$ is the relative velocity between the UAV and the $i$ th waypoint perpendicular to the $X_{R}$ direction.

The complementary equation that describes the relationship between $a_{\sigma}$ and $a$ is given by

$$
a_{\sigma}=a \cos \left(\gamma-\sigma_{i}\right)
$$

By choosing proper $\sigma_{0}$, the angle $\sigma_{i}-\sigma_{0}$ can be made relatively small since the LOS angle variation is small during the flight if a guidance law works properly [29]. In practical flight, the velocity lead angle is also relatively small [30]. With these assumptions in mind, the relative kinematics between the UAV and the $i$ th waypoint can be formulated as

$$
\begin{aligned}
& \dot{y}_{i}=v_{i} \\
& \dot{v}_{i}=-a, \quad i \in\{1,2, \cdots, N\}
\end{aligned}
$$

Define $\boldsymbol{x}=\left[y_{1}, v_{1}, y_{2}, v_{2}, \ldots, y_{N}, v_{N}\right]^{T} \in \mathbb{R}^{2 N \times 1}$ as the system state vector and $\boldsymbol{y}=\left[y_{1}, y_{2}, \ldots, y_{N}\right]^{T} \in \mathbb{R}^{N \times 1}$ as the output vector. Then, the linearized equations of motion can be written in a compact matrix form as

$$
\begin{aligned}
& \dot{x}=A x+B a \\
& y=C x
\end{aligned}
$$

where $\boldsymbol{A} \in \mathbb{R}^{2 N \times 2 N}$ is a block diagonal matrix, $\boldsymbol{B} \in \mathbb{R}^{2 N \times 1}$, and $\boldsymbol{C} \in \mathbb{R}^{1 \times 2 N}$. These three matrices are defined as

$$
\begin{aligned}
& \boldsymbol{A}=\operatorname{diag}\left(\boldsymbol{A}_{1}, \boldsymbol{A}_{2}, \ldots \boldsymbol{A}_{N}\right), \quad \boldsymbol{B}=\left[\boldsymbol{B}_{1}^{T}, \boldsymbol{B}_{2}^{T}, \ldots, \boldsymbol{B}_{N}^{T}\right]^{T}, \quad \boldsymbol{C}=\left[\boldsymbol{C}_{1}, \boldsymbol{C}_{2}, \ldots, \boldsymbol{C}_{N}\right] \\
& \boldsymbol{A}_{i}=\left[\begin{array}{ll}
0 & 1 \\
0 & 0
\end{array}\right], \quad \boldsymbol{B}_{i}=\left[\begin{array}{c}
0 \\
-1
\end{array}\right], \quad \boldsymbol{C}_{i}=\left[\begin{array}{cc}
1 & 0
\end{array}\right], \quad i \in\{1,2, \cdots, N\}
\end{aligned}
$$




\section{Problem Formulation}

In practice, the energy consumption is of paramount importance for a UAV since it determines the endurance of the vehicle. For this reason, this paper considers the following quadratic integral control effort performance index

$$
J=\int_{t}^{t_{f, N}} a^{2}(\tau) d \tau=\left\{\begin{array}{c}
\sum_{i=1}^{N} \int_{t}^{t_{f, i}} a^{2}(\tau) d \tau, \quad t \leq t_{f, 1} \\
\sum_{i=2}^{N} \int_{t}^{t_{f, i}} a^{2}(\tau) d \tau, \quad t_{f, 1}<t \leq t_{f, 2} \\
\vdots \\
\int_{t}^{t_{f, N}} a^{2}(\tau) d \tau, \quad t_{f, N-1}<t \leq t_{f, N}
\end{array}\right.
$$

The aim of this paper is to find analytical solutions of the following two generalized optimal waypoint-following problems:

Problem 1 (Optimal waypoint-following guidance problem) Given the kinematics model (6), finds the guidance command a that minimizes performance index (9) and ensures perfect waypoint passing constraints

$$
y_{i}\left(t_{f, i}\right)=0, \quad i \in\{1,2, \cdots, N\}
$$

Problem 2 (Optimal waypoint-following with partial flight path angle constraint guidance problem) Given the kinematics model (6), finds the guidance command a that satisfies the same conditions as in Problem 1 and the additional M flight path angle constraints

$$
\gamma\left(t_{f, l(j)}\right)=\gamma_{l(j)}^{d}, \quad j \in\{1,2, \cdots, M\}, \quad l(j) \in\{1,2, \cdots, N\}, \quad M \leq N
$$

where $l(j)$ is the index of waypoints that have specific flight path angle constraints and $\gamma_{l(j)}^{d}$ denotes the desired flight angle of the UAV when it passes the $l(j)$ th waypoint.

\section{Guidance Law Design for Optimal Waypoint-Following}

\section{A. Guidance Law Derivation}

This subsection will derive an optimal guidance law to address Problem 1. To reduce the system order, the concept of zero-effort-miss (ZEM) transformation [26, 30] is used in this paper. The ZEM between the UAV and the $i$ th waypoint, 
denoted as $Z_{i}$, is defined as

$$
Z_{i}=\left\{\begin{array}{ll}
C_{i} \Phi_{i}\left(t_{f, i}, t\right)\left[y_{i}, v_{i}\right]^{T}, & t \leq t_{f, i} \\
Z_{i}\left(t_{f, i}\right), & t>t_{f, i}
\end{array}, \quad i \in\{1,2, \ldots, N\}\right.
$$

where $\Phi_{i}\left(t_{f, i}, t\right)$ is the transition matrix associated with matrix $\boldsymbol{A}_{i}$ and is determined as

$$
\Phi\left(t_{f, i}, t\right)=\left[\begin{array}{cc}
1 & t_{f, i}-t \\
0 & 1
\end{array}\right]
$$

Substituting Eq. (13) into Eq. (12) gives the ZEM as

$$
Z_{i}=\left\{\begin{array}{ll}
y_{i}+t_{g o, i} v_{i}, & t \leq t_{f, i} \\
Z_{i}\left(t_{f, i}\right), & t>t_{f, i}
\end{array}, \quad i \in\{1,2, \ldots, N\}\right.
$$

where $t_{g o, i}=t_{f, i}-t$ denotes the remaining flight time, or the so-called time-to-go, to pass the $i$ th waypoint.

The ZEM dynamics can be obtained from Eq. (14) as

$$
\dot{Z}_{i}=\left\{\begin{array}{ll}
-t_{g o, i} a, & t \leq t_{f, i} \\
0, & t>t_{f, i},
\end{array} \quad i \in\{1,2, \ldots, N\}\right.
$$

With this state transformation, the system order now reduces from $2 N$ to $N$. Problem 1 reduces to a problem with the same performance index given by Eq. (9) but reduced-order system dynamics (15). Notice that the ZEM $Z_{i}$ represents the miss distance between the UAV and the $i$ th waypoint if, from the current time onward, the UAV will not apply any maneuver input. With this in mind, the terminal constraints of the new system states are determined as

$$
Z_{i}\left(t_{f, i}\right)=0, \quad i \in\{1,2, \cdots, N\}
$$

According to linear system theory, the solution of system (15) can be obtained as

$$
Z_{i}\left(t_{f, i}\right)-Z_{i}(t)=\int_{t}^{t_{f, i}}-\left(t_{f, i}-\tau\right) a(\tau) d \tau, \quad t \leq t_{f, i}
$$

Imposing the terminal constraints 16 on Eq. 17) gives

$$
Z_{i}(t)=\int_{t}^{t_{f, i}}\left(t_{f, i}-\tau\right) a(\tau) d \tau, \quad t \leq t_{f, i}
$$


Consider the Hilbert space $H=L^{2}\left[t, t_{f, N}\right]$ with the inner product $(f, g)=\int_{t}^{t_{f, i}} f(\tau) g(\tau) d \tau$. According to Lemma 1, shown in Appendix, if the guidance command $a$ is optimal in terms of energy minimization, then there exist $N$ Lagrange multipliers $\lambda_{i}, i \in\{1,2, \cdots, N\}$, such that the lateral acceleration command can be formulated as

$$
a=\left\{\begin{array}{l}
\sum_{i=1}^{N} \lambda_{i}\left(t_{f, i}-t\right), \quad t \leq t_{f, 1} \\
\sum_{i=2}^{N} \lambda_{i}\left(t_{f, i}-t\right), \quad t_{f, 1}<t \leq t_{f, 2} \\
\vdots \\
\lambda_{N}\left(t_{f, N}-t\right), \quad t_{f, N-1}<t \leq t_{f, N}
\end{array}\right.
$$

The Lagrange multipliers $\lambda_{i}$ can then be determined by introducing the expression in Eq. (18) and solving the resultant equations. Note that this approach can be viewed as the extension of Schwarz inequality method [31] to arbitrary number of terminal constraints. Without loss of generality, we only consider the case $t \leq t_{f, 1}$ in the following derivations. By substituting Eq. (19) into Eq. (18) under condition $t \leq t_{f, 1}$, we have

$$
\begin{aligned}
Z_{i}(t) & =\sum_{i^{\prime}=1}^{N} \lambda_{i^{\prime}} \int_{t}^{t_{f, i}}\left(t_{f, i}-\tau\right)\left(t_{f, i^{\prime}}-\tau\right) d \tau \\
& =\sum_{i^{\prime}=1}^{N} \lambda_{i^{\prime}} \int_{t}^{t_{f, \min \left\{i, i^{\prime}\right\}}}\left(t_{f, i}-\tau\right)\left(t_{f, i^{\prime}}-\tau\right) d \tau \\
& =\sum_{i^{\prime}=1}^{N} \lambda_{i^{\prime}} \int_{t}^{t_{f, \min \left\{i, i^{\prime}\right\}}}\left(t_{f, \max \left\{i, i^{\prime}\right\}}-t_{f, \min \left\{i, i^{\prime}\right\}}\right)\left(t_{f, \min \left\{i, i^{\prime}\right\}}-\tau\right)+\left(t_{f, \min \left\{i, i^{\prime}\right\}}-\tau\right)^{2} d \tau \\
& =\sum_{i^{\prime}=1}^{N} \lambda_{i^{\prime}}\left[\frac{t_{g o, \min \left\{i, i^{\prime}\right\}}^{3}}{3}+\frac{t_{g o, \min \left\{i, i^{\prime}\right\}}^{2}}{2}\left(t_{f, \max \left\{i, i^{\prime}\right\}}-t_{f, \min \left\{i, i^{\prime}\right\}}\right)\right] \\
& =\sum_{i^{\prime}=1}^{N} \lambda_{i^{\prime}}\left[\frac{t_{g o, \min \left\{i, i^{\prime}\right\}}^{3}}{3}+\frac{t_{g o, \min \left\{i, i^{\prime}\right\}}^{2}}{2}\left(t_{g o, \max \left\{i, i^{\prime}\right\}}-t_{g o, \min \left\{i, i^{\prime}\right\}}\right)\right] \\
& =\sum_{i^{\prime}=1}^{N} \lambda_{i^{\prime}}\left[\frac{\left.t_{g o, \max \left\{i, i^{\prime}\right\}} t_{g o, \min \left\{i, i^{\prime}\right\}}^{2}-\frac{t_{g o, \min \left\{i, i^{\prime}\right\}}^{3}}{2}\right]}{6}\right]
\end{aligned}
$$

Define $\lambda=\left[\lambda_{1}, \lambda_{2}, \cdots, \lambda_{N}\right]^{T}$ and $\boldsymbol{Z}=\left[Z_{1}, Z_{2}, \cdots, Z_{N}\right]^{T}$ as the Lagrange multiplier vector and the ZEM vector, respectively. Then, Eq. 20, can be rewritten in a compact matrix form as

$$
G \lambda=Z
$$


where $\boldsymbol{G} \in \mathbb{R}^{N \times N}$ is a symmetric matrix, e.g., $\boldsymbol{G}^{T}=\boldsymbol{G}$, and is given by

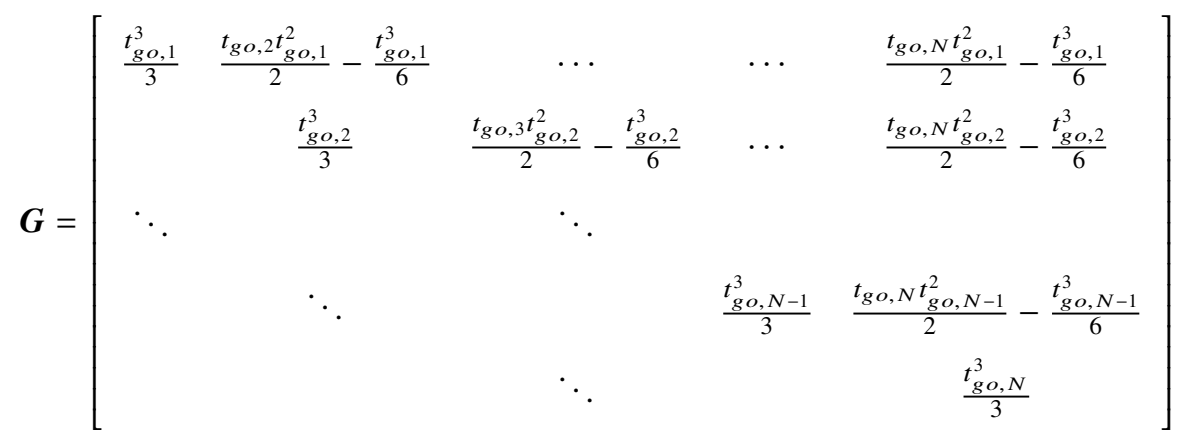

From Eq. 21], the Lagrange multiplier vector $\lambda$ can be obtained as

$$
\lambda=G^{-1} \boldsymbol{Z}
$$

The guidance command for $t \leq t_{f, 1}$ can then be obtained by substituting Eq. [23) into Eq. (19) as

$$
\begin{aligned}
a & =\lambda^{T}\left[t_{g o, 1}, t_{g o, 2}, \ldots, t_{g o, N}\right]^{T} \\
& =\left(\boldsymbol{G}^{-1} \boldsymbol{Z}\right)^{T}\left[t_{g o, 1}, t_{g o, 2}, \ldots, t_{g o, N}\right]^{T}
\end{aligned}
$$

Remark 1 Following similar procedures, the solutions for $t>t_{f, 1}$ can be easily obtained. For example, when $t_{f, 1}<t \leq t_{f, 2}$, the ZEM vector reduces to $\boldsymbol{Z}=\left[Z_{2}, \cdots, Z_{N}\right]^{T}$ and the matrix $\boldsymbol{G}$ becomes

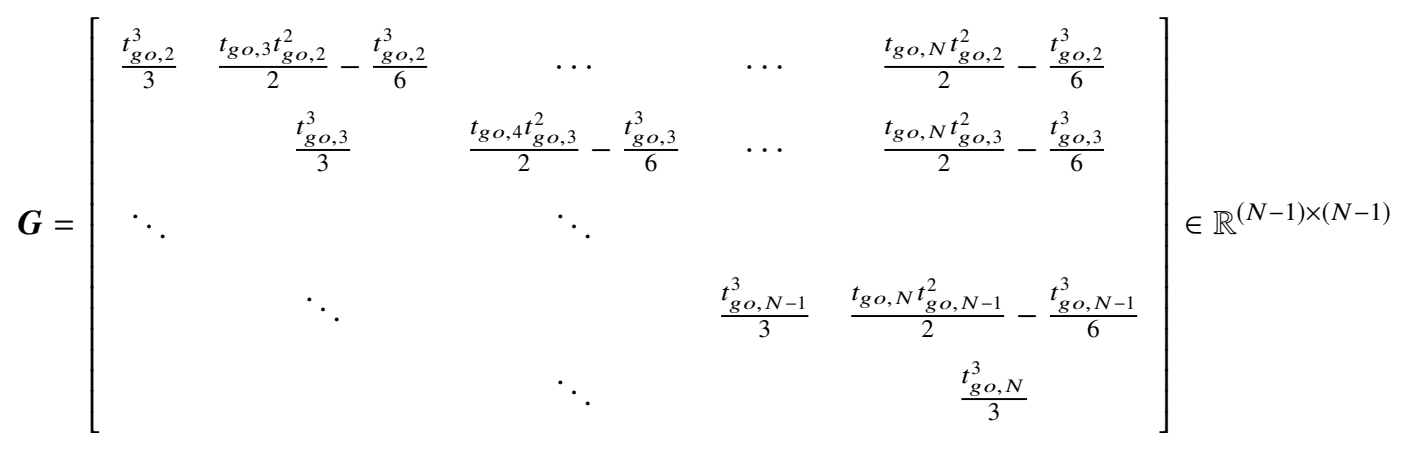

The guidance command for $t_{f, 1}<t \leq t_{f, 2}$ is then given by

$$
a=\left(\boldsymbol{G}^{-1} \boldsymbol{Z}\right)^{T}\left[t_{g o, 2}, t_{g o, 3}, \ldots, t_{g o, N}\right]^{T}
$$

Remark 2 It can be noted that implementing the proposed guidance law requires the knowledge of waypoint passing times $t_{f_{i}}$. Notice that the approximated calculation, shown in Eq. (3), is accurate only when the velocity lead angle is zero, which might not be realistic in practics. For this reason, we update the passing time at every time instant 
as $t_{f, i}=t+t_{g o, i}=t+r_{i} / V$. It is worth pointing out that the approximation error of $t_{g o, i}=r_{i} / V$ decreases as the UAV approaches the ith waypoint. Therefore, accurate passing time can be obtained when the UAV is close to the ith waypoint.

Remark 3 For the purpose of implementation, it is desired to formulate the guidance command in terms of measured signals. Under the assumption that the LOS angle $\sigma_{i}$ and the velocity lead angle $\theta_{i}$ are small, Fig. 1 reveals that

$$
\sigma_{i}-\sigma_{0}=\frac{y_{i}}{r_{i}}
$$

Taking the time derivative of Eq. (27) results in

$$
\begin{aligned}
\dot{\sigma}_{i} & =\frac{v_{i} r_{i}-y_{i} \dot{r}_{i}}{r_{i}^{2}} \\
& =\frac{y_{i} V+v_{i} r_{i}}{r_{i}^{2}} \\
& =\frac{y_{i}+v_{i} t_{g o, i}}{V t_{g o, i}^{2}}
\end{aligned}
$$

Using Eq. (28), the $Z E M Z_{i}$ can then be written in an alternative form as

$$
Z_{i}=V \dot{\sigma}_{i} t_{g o, i}^{2}
$$

Substituting Eq. (29) into Eq. (24) gives the guidance command in terms of measured signals $\dot{\sigma}_{i}, V$ and $r_{i}$. This supports the practical application of the proposed guidance law. Note that although the proposed guidance law is derived based on the linearized engagement kinematics, the error generated in the linearization process can be alleviated by using Eq. (29) in implementation since Eq. (29) transforms the original linear terms into their corresponding nonlinear expressions.

Remark 4 It follows from Eq. (24) that implementing the proposed guidance law requires calculation of the inverse of matrix $\boldsymbol{G}$. Notice that the size of matrix $\boldsymbol{G}$ is proportional to the number of waypoints to be traveled. Therefore, the complexity, or computational burden, of the proposed guidance law increases for a large number of waypoints. However, compared with numerical optimization solutions, the proposed method is still much more efficient since the guidance command is explicitly given as a feedback form of the measured signals.

Remark 5 Although the proposed guidance law is designed based on an ideal autopilot assumption, the algorithm developed can be easily extended to more practical cases with autopilot delays. For example, let us assume that the 
achieved acceleration a through the autopilot is subject to a first-order lag as

$$
\dot{a}=-\frac{1}{\tau_{a}} a+\frac{1}{\tau_{a}} a_{c}
$$

where $\tau_{a}$ denotes the autopilot time constant and $a_{c}$ represents the guidance command generated by the guidance law.

For this first-order lag case, the ZEM $Z_{i}$ is given by [23, 32-34]

$$
Z_{i}=\left\{\begin{array}{ll}
y_{i}+t_{g o, i} v_{i}-a \tau_{a}^{2} \phi\left(\frac{t_{g o, i}}{\tau_{a}}\right), & t \leq t_{f, i} \\
Z_{i}\left(t_{f, i}\right), & t>t_{f, i}
\end{array}, \quad i \in\{1,2, \ldots, N\}\right.
$$

where

$$
\phi(x) \triangleq e^{-x}+x-1
$$

Then, the ZEM dynamics can be readily obtained as

$$
\dot{Z}_{i}=\left\{\begin{array}{ll}
-\tau_{a} \phi\left(\frac{t_{g o, i}}{\tau_{a}}\right) a_{c}, & t \leq t_{f, i} \\
0, & t>t_{f, i}
\end{array} \quad i \in\{1,2, \ldots, N\}\right.
$$

Following similar procedures shown above, we can easily obtain the guidance command $a_{c}$ that can account for the first-order autopilot lag.

\section{B. Particular Cases}

1. $N=1$

When there exists only one waypoint to be visited by the UAV, Problem 1 reduces to energy-optimal intercept problem. For this specific case, the guidance command (19) can be written as

$$
a=\lambda_{1}\left(t_{f, 1}-t\right)
$$

Under condition $N=1$, matrix $\boldsymbol{G}$ reduces to a scalar as $\boldsymbol{G}=t_{g o, 1}^{3} / 3$ and the single Lagrange multiplier can be readily obtained as $\lambda_{1}=3 / t_{g o, 1}^{3} Z_{1}$. Substituting this expression into Eq. 34 gives the explicit guidance command as

$$
a=\frac{3 Z_{1}}{t_{g o, 1}^{2}}
$$

which coincides with the classical optimal PNG. As shown in [35], the PNG with a navigation gain three is energy optimal in the case of single waypoint. However, from the previous derivation, it is clear that simply applying PNG to 
every two consecutive waypoints is not energy optimal when the number of waypoints satisfies $N \geq 2$. The reason is that PNG cannot control the UAV's flight path angle and therefore the energy consumption of PNG is different from that of the proposed guidance law if the arrival angles $\gamma\left(t_{f, i}\right)$ are different.

2. $N=2$

When there are two waypoints to be visited by the UAV, the guidance command (19) under condition $t \leq t_{f, 1}$ can be written as

$$
a=\lambda_{1}\left(t_{f, 1}-t\right)+\lambda_{2}\left(t_{f, 2}-t\right)
$$

and matrix $\boldsymbol{G}$ becomes

$$
\boldsymbol{G}=\left[\begin{array}{cc}
\frac{t_{g o, 1}^{3}}{3} & \frac{t_{g o, 2} t_{g o, 1}^{2}}{2}-\frac{t_{g o, 1}^{3}}{6} \\
\frac{t_{g o, 2} t_{g o, 1}^{2}}{2}-\frac{t_{g o, 1}^{3}}{6} & \frac{t_{g o, 2}^{3}}{3}
\end{array}\right]
$$

The corresponding Lagrange multipliers can be readily obtained from Eq. 23, as

$$
\begin{aligned}
& \lambda_{1}=\frac{6\left(2 t_{g o, 2}^{3} Z_{1}+t_{g o, 1}^{3} Z_{2}-3 t_{g o, 1}^{2} t_{g o, 2} Z_{2}\right)}{t_{g o, 1}^{3}\left(t_{g o, 2}-t_{g o, 1}\right)^{2}\left(4 t_{g o, 2}-t_{g o, 1}\right)} \\
& \lambda_{2}=\frac{6\left(t_{g o, 1} Z_{1}-3 t_{g o, 2} Z_{1}+2 t_{g o, 1} Z_{2}\right)}{t_{g o, 1}\left(t_{g o, 2}-t_{g o, 1}\right)^{2}\left(4 t_{g o, 2}-t_{g o, 1}\right)}
\end{aligned}
$$

Substituting Eq. (38) into Eq. (36) gives the explicit acceleration command as

$$
a=\frac{6\left(2 t_{g o, 2}^{2} Z_{1}-t_{g o, 1} t_{g o, 2} Z_{1}-t_{g o, 1}^{2} Z_{2}\right)}{t_{g o, 1}^{2}\left(t_{g o, 2}-t_{g o, 1}\right)\left(4 t_{g o, 2}-t_{g o, 1}\right)}
$$

Using Eq. 29], the guidance command can be reformulated in terms of measured signals as

$$
a=N_{1} V \dot{\sigma}_{1}+N_{2} V \dot{\sigma}_{2}
$$

where

$$
N_{1}=\frac{-6\left(t_{g o, 1}-2 t_{g o, 2}\right) t_{g o, 2}}{\left(t_{g o, 1}-4 t_{g o, 2}\right)\left(t_{g o, 1}-t_{g o, 2}\right)}, \quad N_{2}=\frac{-6 t_{g o, 2}^{2}}{\left(t_{g o, 1}-4 t_{g o, 2}\right)\left(t_{g o, 1}-t_{g o, 2}\right)}
$$

Define $a_{B}=N_{2} V \dot{\sigma}_{2}$, Eq. (40) can be further reduced to

$$
a=N_{1} V \dot{\sigma}_{1}+a_{B}
$$

which means that the proposed guidance law can be viewed as a biased PNG law with a time-varying navigation gain $N_{1}$ 
when $t \leq t_{f, 1}$.

In the following, we will analyze the effect of the biased term $a_{B}$ to provide better insights of the proposed guidance law. To this end, we assume that the navigation gain $N_{1}$ and the biased term $a_{B}$ are constant in the vicinity of the first waypoint. For PNG with a constant biased term, the closed-form solution is given by [36]

$$
y_{1}=c_{1} t_{g o, 1}^{N_{1}}+c_{2} t_{g o, 1}+\frac{a_{B}}{N_{1}-2} t_{g o, 1}^{2}
$$

where $c_{1}$ and $c_{2}$ are constants determined by the initial conditions.

Since $\sigma_{1}-\sigma_{0} \approx y_{1} / r_{1} \approx y_{1} /\left(V t_{g o, 1}\right)$, we have

$$
\sigma_{1}=\frac{c_{1}}{V} t_{g o, 1}^{N_{1}-1}+\frac{c_{2}}{V}+\frac{a_{B}}{V\left(N_{1}-2\right)} t_{g o, 1}+\sigma_{0}
$$

Differentiating Eq. (44) with respect to time gives the LOS rate $\dot{\sigma}_{1}$ as

$$
\dot{\sigma}_{1}=-\frac{c_{1}}{V}\left(N_{1}-1\right) t_{g o, 1}^{N_{1}-2}-\frac{a_{B}}{V\left(N_{1}-2\right)}
$$

It follows from Eq. 41 that $\lim _{t_{g o, 1} \rightarrow 0} N_{1}=3>2$. Hence, when the UAV approaches the first waypoint, LOS rate $\dot{\sigma}_{1}$ becomes

$$
\lim _{t_{g o, 1} \rightarrow 0^{+}} \dot{\sigma}_{1}=-\frac{a_{B}}{V\left(N_{1}-2\right)}
$$

Therefore, the acceleration command in the vicinity of the first waypoint becomes

$$
\begin{aligned}
\lim _{t_{g o, 1} \rightarrow 0^{+}} a & =-\frac{N_{1}}{N_{1}-2} a_{B}+a_{B} \\
& =-\frac{2}{N_{1}-2} a_{B} \\
& =N^{\prime}{ }_{2} V \dot{\sigma}_{2}
\end{aligned}
$$

where

$$
N_{2}^{\prime}=\frac{12 t_{g o, 2}^{2}}{4 t_{g o, 2}^{2}-2 t_{g o, 1}^{2}+4 t_{g o, 1} t_{g o, 2}}
$$

Since $\lim _{t_{g o, 1} \rightarrow 0^{+}} N_{2}^{\prime} \rightarrow 3$, the guidance command of the proposed guidance law under condition $t_{g o, 1} \rightarrow 0$ reduces to

$$
\lim _{t_{g o, 1} \rightarrow 0^{+}} a=3 V \dot{\sigma}_{2}
$$

which coincides with the guidance command when $t_{f, 1}<t \leq t_{f, 2}$. This clearly reveals that the bias term $N_{2} V \dot{\sigma}_{2}$ helps to reduce the transient effect when the UAV passes the first waypoint. 
3. $N=3$

When there are three waypoints to be visited by the UAV, the guidance command (19) under condition $t \leq t_{f, 1}$ can be written as

$$
a=\lambda_{1}\left(t_{f, 1}-t\right)+\lambda_{2}\left(t_{f, 2}-t\right)+\lambda_{3}\left(t_{f, 3}-t\right)
$$

and matrix $\boldsymbol{G}$ becomes

$$
\boldsymbol{G}=\left[\begin{array}{ccc}
\frac{t_{g o, 1}^{3}}{3} & \frac{t_{g o, 2} t_{g o, 1}^{2}}{2}-\frac{t_{g o, 1}^{3}}{6} & \frac{t_{g o, 3} t_{g o, 1}^{2}}{2}-\frac{t_{g o, 1}^{3}}{6} \\
\frac{t_{g o, 2} t_{g o, 1}^{2}}{2}-\frac{t_{g o, 1}^{3}}{6} & \frac{t_{g o, 2}^{3}}{3} & \frac{t_{g o, 3} t_{g o, 2}^{2}}{2}-\frac{t_{g o, 2}^{3}}{6} \\
\frac{t_{g o, 3} t_{g o, 1}^{2}}{2}-\frac{t_{g o, 1}^{3}}{6} & \frac{t_{g o, 3} t_{g o, 2}^{2}}{2}-\frac{t_{g o, 2}^{3}}{6} & \frac{t_{g o, 3}^{3}}{3}
\end{array}\right]
$$

Following previous derivations, the explicit guidance command is obtained as

$$
\begin{aligned}
a= & \frac{3 t_{g o, 2}\left(t_{g o, 3}-t_{g o, 2}\right)\left[t_{g o, 1}\left(2 t_{g o, 3}+t_{g o, 2}\right)-t_{g o, 2}\left(4 t_{g o, 3}-t_{g o, 2}\right)\right] Z_{1}}{t_{g o, 1}^{2}\left(t_{g o, 1}-t_{g o, 2}\right)\left(t_{g o, 3}-t_{g o, 2}\right)\left(2 t_{g o, 1} t_{g o, 2}+t_{g o, 1} t_{g o, 3}-4 t_{g o, 2} t_{g o, 3}+t_{g o, 2}^{2}\right)} \\
& +\frac{3\left(t_{g o, 3}-t_{g o, 1}\right)\left(2 t_{g o, 3}-t_{g o, 2}-t_{g o, 1}\right) Z_{2}-3\left(t_{g o, 1}-t_{g o, 2}\right)^{2} Z_{3}}{\left(t_{g o, 1}-t_{g o, 2}\right)\left(t_{g o, 3}-t_{g o, 2}\right)\left(2 t_{g o, 1} t_{g o, 2}+t_{g o, 1} t_{g o, 3}-4 t_{g o, 2} t_{g o, 3}+t_{g o, 2}^{2}\right)}
\end{aligned}
$$

Using Eq. 29], the guidance command can be reformulated in terms of measured signals as

$$
a=N_{1} V \dot{\sigma}_{1}+N_{2} V \dot{\sigma}_{2}+N_{3} V \dot{\sigma}_{3}
$$

where

$$
\begin{aligned}
& N_{1}=\frac{3 t_{g o, 2}\left(t_{g o, 3}-t_{g o, 2}\right)\left[t_{g o, 1}\left(2 t_{g o, 3}+t_{g o, 2}\right)-t_{g o, 2}\left(4 t_{g o, 3}-t_{g o, 2}\right)\right]}{\left(t_{g o, 1}-t_{g o, 2}\right)\left(t_{g o, 3}-t_{g o, 2}\right)\left(2 t_{g o, 1} t_{g o, 2}+t_{g o, 1} t_{g o, 3}-4 t_{g o, 2} t_{g o, 3}+t_{g o, 2}^{2}\right)} \\
& N_{2}=\frac{3 t_{g o, 2}^{2}\left(t_{g o, 3}-t_{g o, 1}\right)\left(2 t_{g o, 3}-t_{g o, 2}-t_{g o, 1}\right)}{\left(t_{g o, 1}-t_{g o, 2}\right)\left(t_{g o, 3}-t_{g o, 2}\right)\left(2 t_{g o, 1} t_{g o, 2}+t_{g o, 1} t_{g o, 3}-4 t_{g o, 2} t_{g o, 3}+t_{g o, 2}^{2}\right)} \\
& N_{3}=-\frac{3 t_{g o, 3}^{2}\left(t_{g o, 1}-t_{g o, 2}\right)^{2}}{\left(t_{g o, 1}-t_{g o, 2}\right)\left(t_{g o, 3}-t_{g o, 2}\right)\left(2 t_{g o, 1} t_{g o, 2}+t_{g o, 1} t_{g o, 3}-4 t_{g o, 2} t_{g o, 3}+t_{g o, 2}^{2}\right)}
\end{aligned}
$$

Recall the results derived for $N=2$, the guidance command for $t_{f, 1}<t \leq t_{f, 2}$ is determined as

$$
a=N_{2}^{\prime} V \dot{\sigma}_{2}+N_{3}^{\prime} V \dot{\sigma}_{3}
$$

where

$$
N_{2}^{\prime}=\frac{-6\left(t_{g o, 2}-2 t_{g o, 3}\right) t_{g o, 3}}{\left(t_{g o, 2}-4 t_{g o, 3}\right)\left(t_{g o, 2}-t_{g o, 3}\right)}, \quad N_{3}^{\prime}=\frac{-6 t_{g o, 3}^{2}}{\left(t_{g o, 2}-4 t_{g o, 3}\right)\left(t_{g o, 2}-t_{g o, 3}\right)}
$$


Therefore, the explicit guidance command for $N=3$ is given by

$$
a=\left\{\begin{array}{lr}
N_{1} V \dot{\sigma}_{1}+N_{2} V \dot{\sigma}_{2}+N_{3} V \dot{\sigma}_{3}, & t \leq t_{f, 1} \\
N_{2}^{\prime} V \dot{\sigma}_{2}+N_{3}^{\prime} V \dot{\sigma}_{3}, & t_{f, 1}<t \leq t_{f, 2} \\
3 V \dot{\sigma}_{3}, & t_{f, 2}<t \leq t_{f, 3}
\end{array}\right.
$$

Following similar procedures shown in the previous subsection, the characteristics of the proposed guidance law near the waypoint can also be analyzed in a theoretical way using the concept of biased PNG.

\section{Guidance Law Design for Optimal Waypoint-Following with Partial Flight Path Angle Constraints}

\section{A. Guidance Law Derivation}

In practice, it is desirable to constrain the UAV's flight path angle as some fixed desired values at some certain waypoints for surveillance missions. For this reason, this subsection will derive an optimal guidance law to address Problem 2. With the ZEM state transformation provided in the previous section, Problem 2 reduces to a problem with the same performance index given by Eq. (9) but reduced-order system dynamics (15). The terminal constraints are determined as

$$
\begin{aligned}
& Z_{i}\left(t_{f, i}\right)=0, \quad i \in\{1,2, \cdots, N\} \\
& \gamma\left(t_{f, l(j)}\right)=\gamma_{l(j)}^{d}, \quad j \in\{1,2, \cdots, M\}, \quad l(j) \in\{1,2, \cdots, N\}, \quad M \leq N
\end{aligned}
$$

According to the linear system theory, the solution of ZEM and flight path angle can be obtained as

$$
\begin{aligned}
& Z_{i}\left(t_{f, i}\right)-Z_{i}(t)=\int_{t}^{t_{f, i}}-\left(t_{f, i}-\tau\right) a(\tau) d \tau, \quad t \leq t_{f, i} \\
& \gamma\left(t_{f, l(j)}\right)-\gamma(t)=\int_{t}^{t_{f, l(j)}} \frac{a(\tau)}{V} d \tau, \quad t \leq t_{f, l(j)}
\end{aligned}
$$

Imposing the terminal constraints (58) on Eq. 59] gives

$$
\begin{aligned}
& Z_{i}\left(t_{f, i}\right)=\int_{t}^{t_{f, i}}\left(t_{f, i}-\tau\right) a(\tau) d \tau, \quad t \leq t_{f, i} \\
& \gamma_{l(j)}^{d}-\gamma(t)=\int_{t}^{t_{f, l(j)}} \frac{a(\tau)}{V} d \tau, \quad t \leq t_{f, l(j)}
\end{aligned}
$$

According to Lemma 1, shown in Appendix, if the guidance command $a$ is optimal in terms of energy minimization, then there exist $N+M$ Lagrange multipliers $\lambda_{i}, \beta_{j}, i \in\{1,2, \cdots, N\}, j \in\{1,2, \cdots, M\}$, such that the lateral acceleration 
command can be formulated as

$$
a=a_{\lambda}+a_{\beta}
$$

with

$$
a_{\lambda}=\left\{\begin{array}{c}
\sum_{i=1}^{N} \lambda_{i}\left(t_{f, i}-t\right), \quad t \leq t_{f, 1} \\
\sum_{i=2}^{N} \lambda_{i}\left(t_{f, i}-t\right), \quad t_{f, 1}<t \leq t_{f, 2} \\
\vdots \\
\lambda_{N}\left(t_{f, N}-t\right), \quad t_{f, N-1}<t \leq t_{f, N}
\end{array} \quad a_{\beta}=\left\{\begin{array}{l}
\sum_{j=1}^{M} \frac{\beta_{j}}{V}, \quad t \leq t_{f, l(1)} \\
\sum_{j=2}^{M} \frac{\beta_{j}}{V}, \quad t_{f, l(1)}<t \leq t_{f, l(2)} \\
\vdots \\
\frac{\beta_{M},}{V}, \quad t_{f, l(M-1)}<t \leq t_{f, l(M)}
\end{array}\right.\right.
$$

where $a_{\lambda}$ refers to the ZEM regulation term and $a_{\beta}$ represents the flight path angle error regulation command.

Similar to previous section, we only consider the case $t \leq t_{f, 1}$ in the following derivations. The solutions for $t>t_{f, 1}$ can be easily obtained through similar procedures. Substituting Eq. 61) into Eq. (60) under condition $t \leq t_{f, 1}$ results in

$$
\begin{aligned}
Z_{i}(t) & =\sum_{i^{\prime}=1}^{N} \lambda_{i^{\prime}} \int_{t}^{t_{f, i}}\left(t_{f, i}-\tau\right)\left(t_{f, i^{\prime}}-\tau\right) d \tau+\sum_{j^{\prime}=1}^{M} \frac{\beta_{j^{\prime}}}{V} \int_{t}^{t_{f, i}}\left(t_{f, i}-\tau\right) d \tau \\
& =\sum_{i^{\prime}=1}^{N} \lambda_{i^{\prime}} \int_{t}^{t_{f, \min \left\{i, i^{\prime}\right\}}}\left(t_{f, i}-\tau\right)\left(t_{f, i^{\prime}}-\tau\right) d \tau+\sum_{j^{\prime}=1}^{M} \frac{\beta_{j^{\prime}}}{V} \int_{t}^{t_{f, \min \left\{i, l\left(j^{\prime}\right)\right\}}}\left(t_{f, i}-\tau\right) d \tau \\
\gamma_{l(j)}^{d}-\gamma & =\sum_{i^{\prime}=1}^{N} \frac{\lambda_{i^{\prime}}}{V} \int_{t}^{t_{f, l(j)}}\left(t_{f, i^{\prime}}-\tau\right) d \tau+\sum_{j^{\prime}=1}^{M} \frac{\beta_{j^{\prime}}}{V^{2}} \int_{t}^{t_{f, l(j)}} d \tau \\
& =\sum_{i^{\prime}=1}^{N} \frac{\lambda_{i^{\prime}}}{V} \int_{t}^{t_{f, \min \left\{i^{\prime}, l(j)\right\}}}\left(t_{f, i^{\prime}}-\tau\right) d \tau+\sum_{j^{\prime}=1}^{M} \frac{\beta_{j^{\prime}}}{V^{2}} \int_{t}^{t_{f, \min \left\{l(j), l\left(j^{\prime}\right)\right\}}} d \tau
\end{aligned}
$$

Evaluating the integrals in Eqs. 63) and 64) gives

$$
\begin{aligned}
\int_{t}^{t_{f, \min \left\{i, i^{\prime}\right\}}}\left(t_{f, i}-\tau\right)\left(t_{f, i^{\prime}}-\tau\right) d \tau \\
=\int_{t}^{t_{f, \min \left\{i, i^{\prime}\right\}}}\left(t_{f, \max \left\{i, i^{\prime}\right\}}-t_{f, \min \left\{i, i^{\prime}\right\}}\right)\left(t_{f, \min \left\{i, i^{\prime}\right\}}-\tau\right)+\left(t_{f, \min \left\{i, i^{\prime}\right\}}-\tau\right)^{2} d \tau \\
=\frac{t_{g o, \min \left\{i, i^{\prime}\right\}}^{3}}{3}+\frac{t_{g o, \min \left\{i, i^{\prime}\right\}}^{2}}{2}\left(t_{f, \max \left\{i, i^{\prime}\right\}}-t_{f, \min \left\{i, i^{\prime}\right\}}\right) \\
=\frac{t_{g o, \min \left\{i, i^{\prime}\right\}}^{3}+\frac{t_{g o, \min \left\{i, i^{\prime}\right\}}^{2}}{2}\left(t_{g o, \max \left\{i, i^{\prime}\right\}}-t_{g o, \min \left\{i, i^{\prime}\right\}}\right)}{3}=\frac{t_{g o, \max \left\{i, i^{\prime}\right\}} t_{g o, \min \left\{i, i^{\prime}\right\}}^{2}-\frac{t_{g o, \min \left\{i, i^{\prime}\right\}}^{3}}{2}}{2}
\end{aligned}
$$




$$
\begin{aligned}
& \int_{t}^{t_{f, \min \left\{i, l\left(j^{\prime}\right)\right\}}}\left(t_{f, i}-\tau\right) d \tau= \begin{cases}\int_{t}^{t_{f, i}}\left(t_{f, i}-\tau\right) d \tau, & i<l\left(j^{\prime}\right) \\
\int_{t}^{t_{f, l\left(j^{\prime}\right)}}\left(t_{f, l\left(j^{\prime}\right)}-\tau+t_{f, i}-t_{f, l\left(j^{\prime}\right)}\right) d \tau, & i \geq l\left(j^{\prime}\right)\end{cases} \\
& = \begin{cases}\frac{t_{g o, i}^{2}}{2}, & i<l\left(j^{\prime}\right) \\
t_{g o, i} t_{g o, l\left(j^{\prime}\right)}-\frac{t_{g o, l\left(j^{\prime}\right)}^{2},}{2}, & i \geq l\left(j^{\prime}\right)\end{cases} \\
& \int_{t}^{t_{f, \min \left\{i^{\prime}, l(j)\right\}}}\left(t_{f, i^{\prime}}-\tau\right) d \tau= \begin{cases}\int_{t}^{t_{f, i^{\prime}}}\left(t_{f, i^{\prime}}-\tau\right) d \tau, & i^{\prime}<l(j) \\
\int_{t}^{t_{f, l(j)}}\left(t_{f, l(j)}-\tau+t_{f, i^{\prime}}-t_{f, l(j)}\right) d \tau, & i^{\prime} \geq l(j)\end{cases} \\
& = \begin{cases}\frac{t_{g o, i^{\prime}}^{2}}{2}, & i^{\prime}<l(j) \\
t_{g o, i^{\prime}} t_{g o, l(j)}-\frac{t_{g o, l(j)}^{2}}{2}, & i^{\prime} \geq l(j)\end{cases} \\
& \int_{t}^{t_{f, \min \left\{l(j), l\left(j^{\prime}\right)\right\}}} d \tau=t_{g o, \min \left\{l(j), l\left(j^{\prime}\right)\right\}}
\end{aligned}
$$

Define $\boldsymbol{\beta}=\left[\beta_{1}, \beta_{2}, \cdots, \beta_{M}\right]^{T}$ and $\boldsymbol{e}_{\gamma}=\left[\gamma_{l(1)}^{d}-\gamma, \gamma_{l(2)}^{d}-\gamma, \cdots, \gamma_{l(M)}^{d}-\gamma\right]^{T}$ as the Lagrange multiplier vector and the flight path angle error vector, respectively. Substituting Eqs. 655-680 into Eqs. 63) and 646 yields

$$
\boldsymbol{G}\left[\begin{array}{c}
\lambda \\
\boldsymbol{\beta}
\end{array}\right]=\left[\begin{array}{c}
\boldsymbol{Z} \\
\boldsymbol{e}_{\gamma}
\end{array}\right], \quad \boldsymbol{G}=\left[\begin{array}{cc}
\boldsymbol{G}_{1} & \boldsymbol{G}_{12} \\
\boldsymbol{G}_{21} & \boldsymbol{G}_{2}
\end{array}\right]
$$

where $\boldsymbol{G}_{1} \in \mathbb{R}^{N \times N}$ is a symmetric matrix which is given by

$$
\boldsymbol{G}_{1}=\left[\begin{array}{ccccc}
\frac{t_{g o, 1}^{3}}{3} & \frac{t_{g o, 2} t_{g o, 1}^{2}}{2}-\frac{t_{g o, 1}^{3}}{6} & \ldots & \ldots & \frac{t_{g o, N} t_{g o, 1}^{2}}{2}-\frac{t_{g o, 1}^{3}}{6} \\
\frac{t_{g o, 2}^{3}}{3} & \frac{t_{g o, 3} t_{g o, 2}^{2}}{2}-\frac{t_{g o, 2}^{3}}{6} & \ldots & \frac{t_{g o, N} t_{g o, 2}^{2}}{2}-\frac{t_{g o, 2}^{3}}{6} \\
\ddots & \ddots & \frac{t_{g o, N-1}^{3}}{3} & \frac{t_{g o, N} t_{g o, N-1}^{2}}{2}-\frac{t_{g o, N-1}^{3}}{6} \\
& \ddots & \ddots & & \frac{t_{g o, N}^{3}}{3}
\end{array}\right]
$$

and $\boldsymbol{G}_{12}=\boldsymbol{G}_{21}^{T}=\left(g_{i j}\right) \in \mathbb{R}^{N \times M}$ with elements $g_{i j}$ being

$$
g_{i j}= \begin{cases}\frac{t_{g o, i}^{2}}{2 V}, & i<l(j) \\ \frac{t_{g o, i} t_{g o, l(j)}}{V}-\frac{t_{g o, l(j)}^{2}}{2 V}, & i \geq l(j)\end{cases}
$$


and $\boldsymbol{G}_{2} \in \mathbb{R}^{M \times M}$ is a symmetric matrix which is obtained as

$$
\boldsymbol{G}_{2}=\frac{1}{V^{2}}\left[\begin{array}{ccccc}
t_{g o, l(1)} & t_{g o, l(1)} & \ldots & \ldots & t_{g o, l(1)} \\
& t_{g o, l(2)} & t_{g o, l(2)} & \ldots & t_{g o, l(2)} \\
\ddots & & \ddots & & \\
& \ddots & & t_{g o, l(M-1)} & t_{g o, l(M-1)} \\
& & \ddots & & t_{g o, l(M)}
\end{array}\right]
$$

From Eq. 69, the Lagrange multiplier vectors $\lambda$ and $\beta$ can be obtained as

$$
\left[\begin{array}{l}
\lambda \\
\beta
\end{array}\right]=G^{-1}\left[\begin{array}{l}
\boldsymbol{Z} \\
\boldsymbol{e}_{\gamma}
\end{array}\right]
$$

The explicit guidance command for $t \leq t_{f, 1}$ can then be readily obtained by substituting Eq. (73) into Eq. (61) as

$$
\begin{aligned}
a & =\lambda^{T}\left[t_{g o, 1}, t_{g o, 2}, \ldots, t_{g o, N}\right]^{T}+\boldsymbol{\beta}^{T} \underbrace{\left[\frac{1}{V}, \frac{1}{V}, \ldots, \frac{1}{V}\right]^{T}}_{M \text { elements }} \\
& =\left(\boldsymbol{G}^{-1}\left[\begin{array}{c}
\boldsymbol{Z} \\
\boldsymbol{e}_{\gamma}
\end{array}\right]\right)^{T}[t_{g o, 1}, t_{g o, 2}, \ldots, t_{g o, N}, \underbrace{\frac{1}{V}, \frac{1}{V}, \ldots, \frac{1}{V}}_{M \text { elements }}]^{T}
\end{aligned}
$$

Remark 6 From Eq. (74), it is clear that the implementation of the proposed guidance law requires the inverse of matrix $\boldsymbol{G}$. Since matrix $\boldsymbol{G}$ is defined as a block form, the block inverse approach [37] can be applied to simplify the matrix inverse calculation as

$$
\boldsymbol{G}^{-1}=\left[\begin{array}{cc}
\left(\boldsymbol{G}_{1}-\boldsymbol{G}_{12} \boldsymbol{G}_{2}^{-1} \boldsymbol{G}_{12}^{T}\right)^{-1} & -\boldsymbol{G}_{1}^{-1} \boldsymbol{G}_{12}\left(\boldsymbol{G}_{2}-\boldsymbol{G}_{12} \boldsymbol{G}_{1}^{-1} \boldsymbol{G}_{12}^{T}\right)^{-1} \\
-\left(\boldsymbol{G}_{2}-\boldsymbol{G}_{12} \boldsymbol{G}_{1}^{-1} \boldsymbol{G}_{12}^{T}\right)^{-1} \boldsymbol{G}_{12}^{T} \boldsymbol{G}_{1}^{-1} & \left(\boldsymbol{G}_{2}-\boldsymbol{G}_{12} \boldsymbol{G}_{1}^{-1} \boldsymbol{G}_{12}^{T}\right)^{-1}
\end{array}\right]
$$

Remark 7 Note that the proposed guidance law is generic. For this reason, it can be applied to general UAV waypoint-following guidance missions with an arbitrary number of waypoints and an arbitrary number of arrival angle constraints. However, the computational burden increases with the increase in the number of boundary constraints. 


\section{B. Particular Case: $M=N$}

Let us consider the case that the UAV is required to pass multiple waypoints and every waypoint requires a specific arrival angle $\gamma_{i}^{d}$. For this specific case, the proposed guidance law is equivalent to TSG applied to every two consecutive waypoints. To validate this, let $a_{i}$ and $\bar{a}_{i}$ be, respectively, the guidance commands of the proposed guidance law and TSG between the $(i-1)$ th and the $i$ th waypoints, with $i=0$ being the initial point for convenience. The corresponding energy consumptions of these two different guidance laws between the $(i-1)$ th and the $i$ th waypoints are then determined as

$$
\begin{aligned}
& J_{i}=\int_{t_{f, i}}^{t_{f, i+1}} a_{i}^{2}(\tau) d \tau \\
& \bar{J}_{i}=\int_{t_{f, i}}^{t_{f, i+1}} \bar{a}_{i}^{2}(\tau) d \tau
\end{aligned}
$$

The equivalence of these two guidance laws will be proved through mathematical induction. When there exists only one waypoint to be visited by the UAV with a specified flight path, e.g., $N=1$, Problem 2 reduces to energy-optimal rendezvous problem. Then, the guidance command of the proposed guidance law, shown in Eq. [61, can be formulated as

$$
a=\lambda_{1}\left(t_{f, 1}-t\right)+\frac{\beta_{1}}{V}
$$

Note that matrix $\boldsymbol{G}$ under condition $N=1$ becomes

$$
\boldsymbol{G}=\left[\begin{array}{cc}
\frac{t_{g o, 1}^{3}}{3} & \frac{t_{g o, 1}^{2}}{2 V} \\
\frac{t_{g o, 1}^{2}}{2 V} & \frac{t_{g o, 1}}{V^{2}}
\end{array}\right]
$$

From Eq. 73, we can readily solve the Lagrange multipliers as

$$
\left[\begin{array}{l}
\lambda_{1} \\
\beta_{1}
\end{array}\right]=\frac{12 V^{2}}{t_{g o, 1}^{3}}\left[\begin{array}{c}
\frac{Z_{1}}{V^{2}}-\frac{t_{g o, 1}}{2 V}\left(\gamma_{1}^{d}-\gamma\right) \\
-\frac{t_{g o, 1}}{2 V} Z_{1}+\frac{t_{g o, 1}^{2}}{3}\left(\gamma_{1}^{d}-\gamma\right)
\end{array}\right]
$$

Substituting Eq. (79) into Eq. (77) gives the explicit guidance command as

$$
a=\frac{6 Z_{1}}{t_{g o, 1}^{2}}-\frac{2 V}{t_{g o, 1}}\left(\gamma_{1}^{d}-\gamma\right)
$$

which coincides with the classical TSG. As shown in [35], the TSG is energy optimal in the case of single waypoint with an arrival angle constraint. 
Table 1 Inertial positions of all waypoints.

\begin{tabular}{cc}
\hline \hline Waypoint ID & Inertial position \\
\hline 1 & $(1000 m, 500 m)$ \\
2 & $(2000 m, 750 m)$ \\
3 & $(2500 m, 1000 m)$ \\
4 & $(4000 m, 1500 m)$ \\
5 & $(6000 m, 2000 m)$ \\
6 & $(7500 m, 1500 m)$ \\
7 & $(9000 m, 1000 m)$ \\
8 & $(11000 m, 0 m)$ \\
\hline \hline
\end{tabular}

By the mathematical induction concept, assume that for $N=L>1$, we have

$$
a_{L}=\bar{a}_{L}, \quad J_{L}=\bar{J}_{L}
$$

which means that both guidance laws ensure the same flight path at the $L$ th waypoint.

Then, for $N=L+1$, if the guidance command of TSG is different from the proposed guidance law, e.g., $a_{L+1} \neq \bar{a}_{L+1}$, we have $J_{L+1}<\bar{J}_{L+1}$ since the proposed guidance law guarantees global optimality. Notice that both guidance laws have the same boundary constraints at the $(L+1)$ th waypoint. This means that condition $a_{L+1} \neq \bar{a}_{L+1}$ violates the optimality of TSG. Therefore, we have $a_{L+1}=\bar{a}_{L+1}$ and consequently these two guidance laws provide the same level of optimality of energy consumption when $M=N$. However, for the problem with $M<N$, TSG is not applicable and applying hybrid PN/TSG to every two consecutive waypoints is obviously not energy optimal.

\section{Numerical Simulations}

In this section, nonlinear numerical simulations are performed to validate the proposed guidance law. In the considered scenario, a UAV with constant speed $V=30 \mathrm{~m} / \mathrm{s}$ is required to follow 8 waypoints. The UAV initially locates at $(0 \mathrm{~m}, 0 \mathrm{~m})$ with an initial flight path angle of $30^{\circ}$. The inertial positions of all waypoints are summarized in Table 1 .

\section{A. Performance of Guidance Law 24)}

This subsection considers the problem of minimum effort waypoint following without any arrival angle constraint to validate the effectiveness of guidance law 24]. For the purpose of comparison, the optimal PNG is also applied to every two consecutive waypoints in the simulations. Fig. 2 (a) compares the UAV flight trajectories obtained from these two different guidance laws. From this figure, it is clear that both guidance laws can successfully drive the UAV to follow the desired waypoints. The maximum recorded miss distance between the UAV and the waypoint under both guidance laws is less than $0.1 \mathrm{~m}$ in the simulations. The flight path angle response comparisons are shown in Fig. 2(b). As shown 


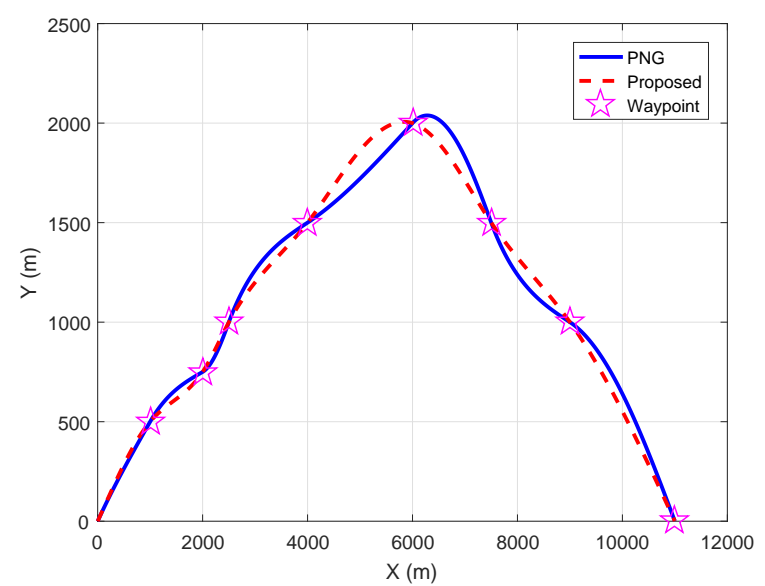

(a)

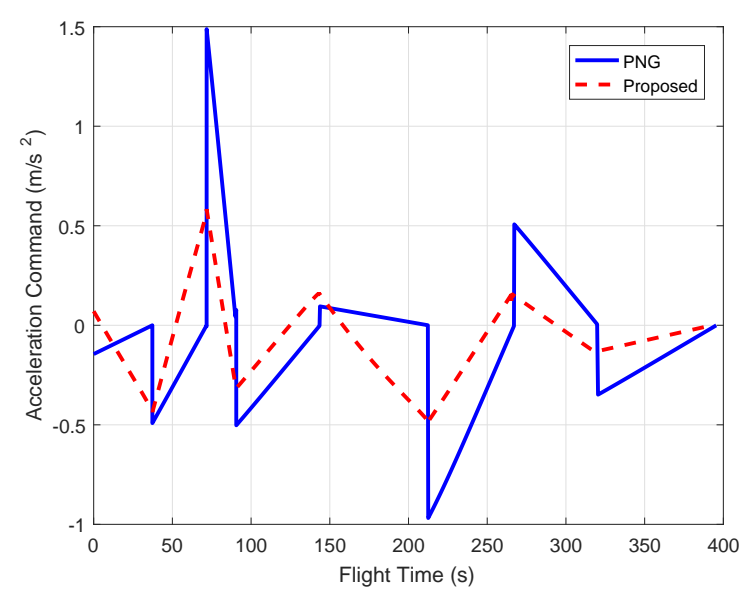

(c)

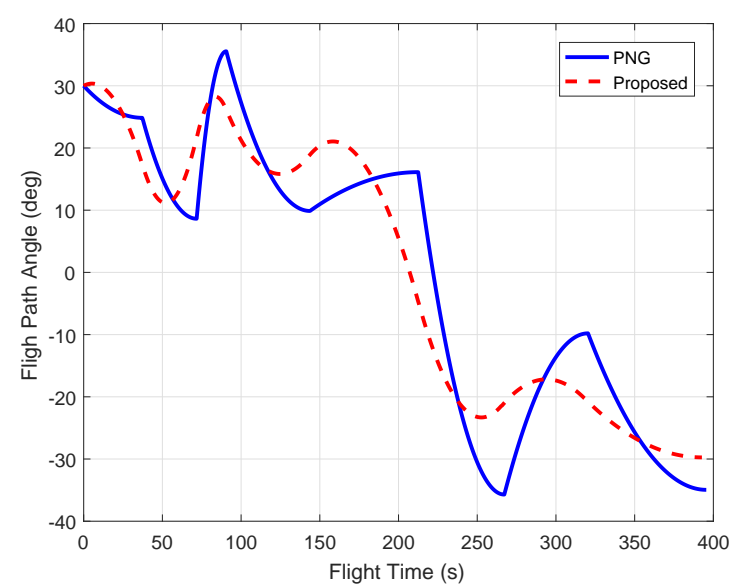

(b)

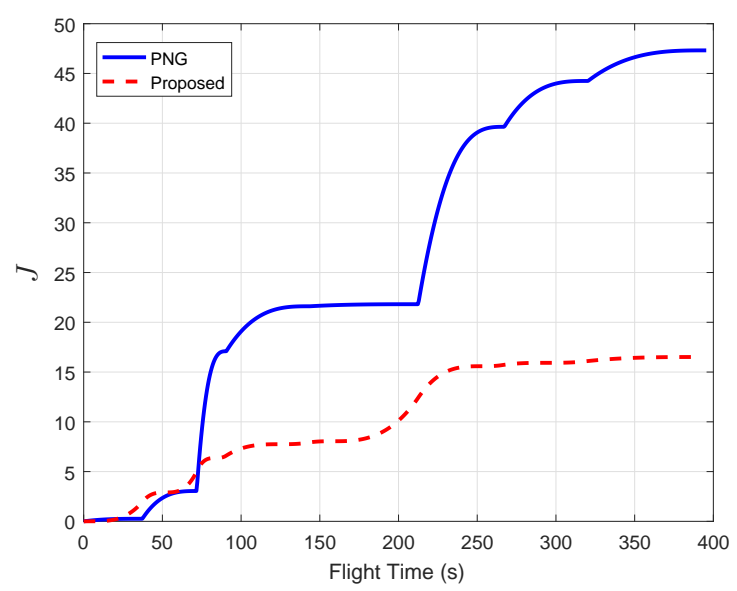

(d)

Fig. 2 Comaprison results without angle constraint. (a) Flight trajectory. (b) Flight path angle. (c) Acceleration command. (d) Performance index

in this figure, the UAV guided by PNG shows sharp turns when it passes waypoints while the proposed guidance law ensures a smooth trajectory.

The acceleration commands of different guidance laws are presented in Fig. 2 (c), which shows that the guidance command under PNG exhibits discontinuity when passing waypoints. As a comparison, the proposed optimal guidance law generates a continuous command, which is more desirable for the implementation. The quantitative comparison results of energy consumption obtained from both guidance laws are summarized in Fig. 2(d). From this figure, we can clearly observe that the proposed guidance law requires less energy consumption, compared with PNG. In the considered scenario, the proposed guidance law helps to reduce more than $40 \%$ energy consumption. Therefore, the UAV guided by the proposed approach is expected to have longer endurance than PNG. 


\section{B. Performance of Guidance Law (74)}

This subsection considers the problem of minimum effort waypoint following with partial arrival angle constraint to validate the effectiveness of guidance law (74). In the scenario considered, we assume that the UAV is required to pass the 4 th waypoint with desired flight path angle $0^{\circ}$ and the 8 th waypoint with desired flight path angle $-90^{\circ}$. For comparison, the optimal guidance law (OGL) is also applied to every two consecutive waypoints in the simulations. The OGL considered here is defined as

$$
\mathrm{OGL}= \begin{cases}\text { TSG, } & \text { if next waypoint requires specific arrival angle } \\ \text { PNG, } & \text { Otherwise }\end{cases}
$$

The UAV flight trajectories and flight path angle profiles obtained from OGL as well as the proposed guidance law are shown in Figs. 3 (a) and 3 (b), respectively. These two figures reveal that the UAV guided by both guidance laws can successfully accomplish its waypoint following mission. The recorded miss distance and flight path angle error obtained from both guidance laws are less than $0.1 \mathrm{~m}$ and $0.5^{\circ}$ in our simulations. Compare with previous scenario without any arrival angle constraint, PNG generates the same trajectory for the first three waypoints. On the other hand, the UAV flight trajectory under the proposed guidance law shows slight difference since the additional angle regulation term $a_{\beta}$ starts to work from the beginning.

Fig. 3 (c) compares the UAV acceleration command generated by these two different guidance laws. Unlike the previous scenario, the proposed guidance law also shows discontinuity when the UAV passes a waypoint that requires a specific arrival angle, e.g., the 4th waypoint in the considered scenario. The reason for this fact is that the angle regulation term $\beta_{1} / V$ disappears when $t>t_{f, 4}$. After passing the 7 th waypoint, there exists only one waypoint, i.e., the 8th, to be visited with a specific arrival angle constraint. For this reason, the proposed guidance law reduces to classical TSG when $t>t_{f, 7}$. Therefore, the proposed guidance law and OGL are expected to share similar characteristics during that period, which is confirmed by Fig. 3 (c). The quantitative comparison results of energy consumption obtained from both guidance laws are presented in Fig. 3(d). From this figure, it can be noted that the proposed guidance requires approximate $20 \%$ less energy consumption in the considered scenario, compared with OGL. For this reason, the proposed guidance law can be utilized to increase the endurance of the UAV.

\section{Conclusions}

This paper discusses the minimum-effort waypoint-following guidance problem for a UAV. Both optimal waypointfollowing guidance and optimal waypoint-following guidance with partial flight path angle constraint are rigorously derived using optimal control theory. When there exits only one waypoint to be traveled by the UAV, the proposed guidance laws reduce to the well-known PNG and TSG. The guidance law developed is generic and can be applied to an 


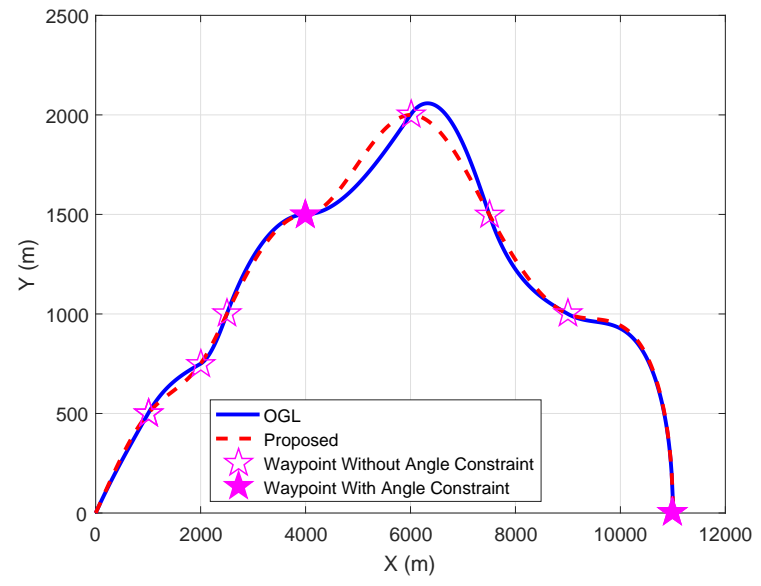

(a)

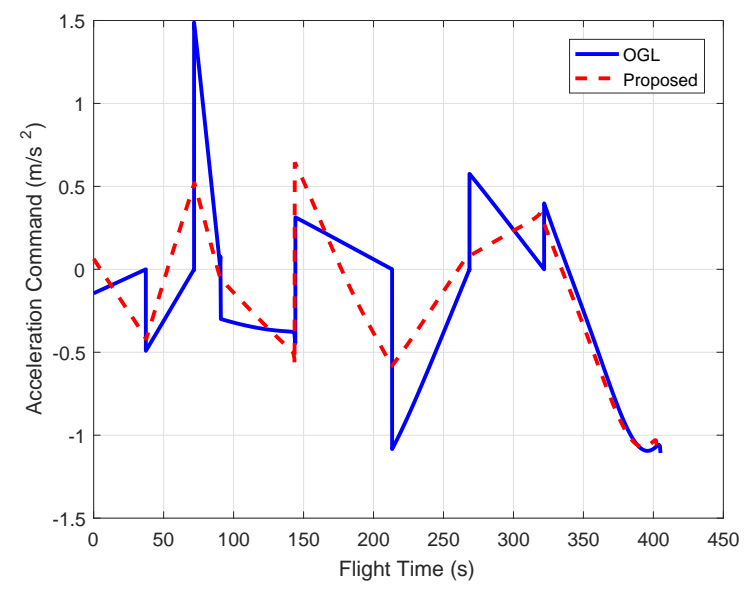

(c)

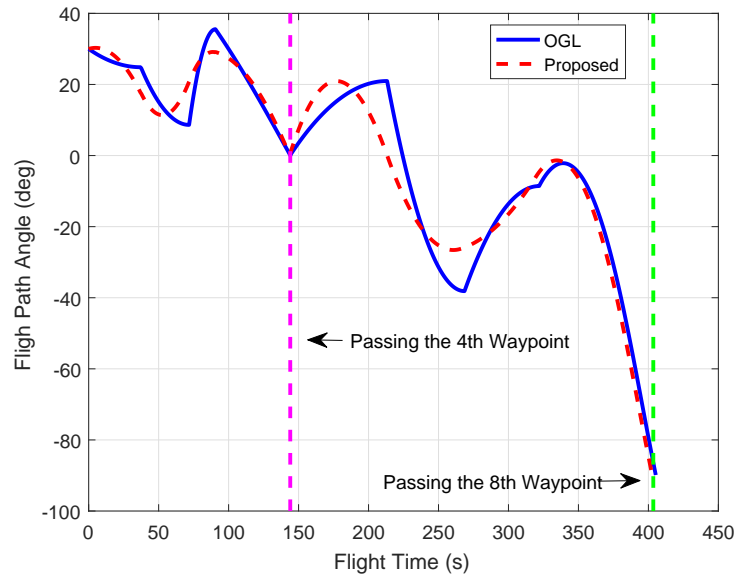

(b)

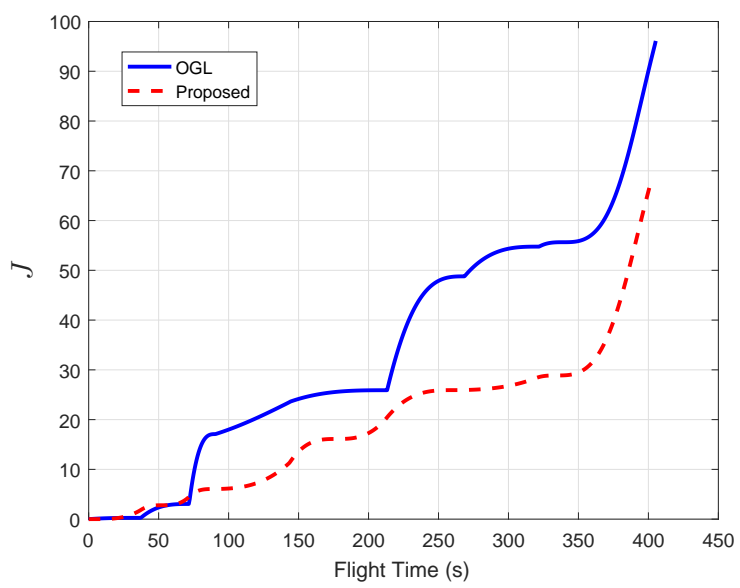

(d)

Fig. 3 Comaprison results with angle constraint. (a) Flight trajectory. (b) Flight path angle. (c) Acceleration command. (d) Performance index 
arbitrary number of waypoints with/without an arbitrary number of arrival angle constraints. Although the proposed guidance law is derived based on a linear kinematics model, nonlinear numerical simulations clearly validate the effectiveness of the proposed guidance law.

By exploiting the advantages of the proposed approach, the guidance law developed in this paper can also be applied to midcourse guidance of sea-skimming anti-ship missiles and cruise missiles.

\section{Appendix: Lemma 1}

This appendix collects a useful lemma from [38] that has been utilized in the derivation of the proposed guidance laws.

Lemma 1 Let $H$ be a Hilbert space and $\boldsymbol{\alpha}_{1}, \boldsymbol{\alpha}_{2}, \cdots, \boldsymbol{\alpha}_{n}$ be a set of $n$ linearly independent vectors in $H$. If the condition $\left(\boldsymbol{x}, \boldsymbol{\alpha}_{i}\right)=c_{i}, i \in\{1,2, \cdots, n\}$, with $c_{i}$ being arbitrary scalars, holds among all vectors of $H$, then, the one that has the minimum norm is given by

$$
\boldsymbol{x}_{\min }=\sum_{i=1}^{n} b_{i} \alpha_{i}
$$

where the coefficients $b_{i}$ satisfy the condition

$$
\sum_{i=1}^{n}\left(\alpha_{i}, \alpha_{j}\right)=b_{j}, \quad j \in\{1,2, \cdots, n\}
$$

\section{References}

[1] Xargay, E., Kaminer, I., Pascoal, A., Hovakimyan, N., Dobrokhodov, V., Cichella, V., Aguiar, A., and Ghabcheloo, R., "Time-critical cooperative path following of multiple unmanned aerial vehicles over time-varying networks," Journal of Guidance, Control, and Dynamics, Vol. 36, No. 2, 2013, pp. 499-516. doi:10.2514/1.56538.

[2] Yu, M.-J., Atkins, E. M., Kolmanovsky, I., and Bernstein, D., "Trim-commanded adaptive control for waypoint-defined trajectory following," AIAA Guidance, Navigation, and Control (GNC) Conference, 2013. doi:10.2514/6.2013-5000.

[3] Kaminer, I., Pascoal, A., Xargay, E., Hovakimyan, N., Cao, C., and Dobrokhodov, V., "Path following for small unmanned aerial vehicles using L1 adaptive augmentation of commercial autopilots," Journal of Guidance, Control, and Dynamics, Vol. 33, No. 2, 2010, pp. 550-564. doi:10.2514/1.42056.

[4] Innocenti, M., Pollini, L., and Turra, D., "Guidance of unmanned air vehicles based on fuzzy sets and fixed waypoints," Journal of Guidance, Control, and Dynamics, Vol. 27, No. 4, 2004, pp. 715-720. doi:10.2514/1.2651.

[5] Tsourdos, A., White, B., and Shanmugavel, M., Cooperative path planning of unmanned aerial vehicles, John Wiley \& Sons, 2010. 
[6] Park, S., Deyst, J., and How, J., "A new nonlinear guidance logic for trajectory tracking," AIAA Guidance, Navigation, and Control Conference and Exhibit, 2004. doi:10.2514/6.2004-4900.

[7] Park, S., Deyst, J., and How, J. P., "Performance and lyapunov stability of a nonlinear path following guidance method," Journal of Guidance, Control, and Dynamics, Vol. 30, No. 6, 2007, pp. 1718-1728. doi:10.2514/1.28957.

[8] B. Medagoda, E. D., and Gibbens, P. W., "Synthetic-waypoint guidance algorithm for following a desired flight trajectory," Journal of Guidance, Control, and Dynamics, Vol. 33, No. 2, 2010, pp. 601-606. doi:10.2514/1.46204.

[9] Nelson, D. R., Barber, D. B., McLain, T. W., and Beard, R. W., "Vector field path following for small unmanned air vehicles," American Control Conference, 2006, IEEE, 2006, pp. 7-pp. doi:10.1109/ACC.2006.1657648.

[10] Frew, E. W., Langelaan, J., and Joo, S., “Adaptive receding horizon control for vision-based navigation of small unmanned aircraft," American Control Conference, 2006, IEEE, 2006, pp. 6-pp. doi:10.1109/ACC.2006.1656539.

[11] Keviczky, T., and Balas, G. J., "Flight test of a receding horizon controller for autonomous UAV guidance," American Control Conference, 2005. Proceedings of the 2005, IEEE, 2005, pp. 3518-3523. doi:10.1109/ACC.2005.1470518.

[12] Sprinkle, J., Eklund, J. M., Kim, H. J., and Sastry, S., "Encoding aerial pursuit/evasion games with fixed wing aircraft into a nonlinear model predictive tracking controller," Decision and Control, 2004. CDC. 43rd IEEE Conference on, Vol. 3, IEEE, 2004, pp. 2609-2614. doi:10.1109/CDC.2004.1428851.

[13] Ratnoo, A., Hayoun, S., Granot, A., and Shima, T. Y., "Path following using trajectory shaping guidance," AIAA Guidance, Navigation, and Control (GNC) Conference, 2013. doi:10.2514/6.2013-5233.

[14] Ratnoo, A., Hayoun, S. Y., Granot, A., and Shima, T., "Path Following Using Trajectory Shaping Guidance," Journal of Guidance, Control, and Dynamics, Vol. 38, No. 1, 2014, pp. 106-116. doi:10.2514/1.G000300.

[15] Yamasaki, T., Balakrishnan, S., and Takano, H., "Separate-channel integrated guidance and autopilot for automatic pathfollowing," Journal of Guidance, Control, and Dynamics, Vol. 36, No. 1, 2012, pp. 25-34. doi:10.2514/1.58259.

[16] Cho, N., Kim, Y., and Park, S., “Three-dimensional nonlinear differential geometric path-following guidance law,” Journal of Guidance, Control, and Dynamics, Vol. 38, No. 12, 2015, pp. 2366-2385. doi:10.2514/1.G001060.

[17] Manyam, S. G., Rathinam, S., Casbeer, D., and Garcia, E., "Tightly bounding the shortest dubins paths through a sequence of points," Journal of Intelligent \& Robotic Systems, Vol. 88, No. 2-4, 2017, pp. 495-511. doi:10.1007/s10846-016-0459-4.

[18] Manyam, S., Rathinam, S., and Casbeer, D., "Dubins paths through a sequence of points: Lower and upper bounds," Unmanned Aircraft Systems (ICUAS), 2016 International Conference on, IEEE, 2016, pp. 284-291. doi:10.1109/ICUAS.2016.7502623.

[19] Whang, I. H., and Hwang, T. W., "Horizontal waypoint guidance design using optimal control," IEEE Transactions on Aerospace and Electronic Systems, Vol. 38, No. 3, 2002, pp. 1116-1120. doi:10.1109/TAES.2002.1039430. 
[20] Spangelo, I., and Egeland, O., "Generation of energy-optimal trajectories for an autonomous underwater vehicle," Robotics and Automation, 1992. Proceedings., 1992 IEEE International Conference on, IEEE, 1992, pp. 2107-2112. doi:10.1109/ROBOT. 1992.219970.

[21] Moon, G., and Kim, Y., "Optimum flight path design passing through waypoints for autonomous flight control system," AIAA Guidance, Navigation, and Control Conference and Exhibit, 2003. doi:10.2514/6.2003-5334.

[22] Ryoo, C.-K., Shin, H.-S., and Tahk, M.-J., "Energy optimal waypoint guidance synthesis for antiship missiles," IEEE Transactions on Aerospace and Electronic Systems, Vol. 46, No. 1. doi:10.1109/TAES.2010.5417149.

[23] Cottrell, R. G., “Optimal intercept guidance for short-range tactical missiles,” AIAA Journal, Vol. 9, No. 7, 1971, pp. 1414-1415. doi:10.2514/3.6369.

[24] Jeon, I.-S., and Lee, J.-I., “Optimality of proportional navigation based on nonlinear formulation,” IEEE Transactions on Aerospace and Electronic Systems, Vol. 46, No. 4, 2010, pp. 2051-2055. doi:10.1109/TAES.2010.5595614.

[25] Palumbo, N. F., Blauwkamp, R. A., and Lloyd, J. M., "Basic principles of homing guidance," Johns Hopkins APL Technical Digest, Vol. 29, No. 1, 2010, pp. 25-41.

[26] Shaferman, V., and Shima, T., "Linear quadratic guidance laws for imposing a terminal intercept angle," Journal of Guidance, Control, and Dynamics, Vol. 31, No. 5, 2008, pp. 1400-1412. doi:10.2514/1.32836.

[27] Palumbo, N. F., Blauwkamp, R. A., and Lloyd, J. M., "Modern homing missile guidance theory and techniques," Johns Hopkins APL technical digest, Vol. 29, No. 1, 2010, pp. 42-59.

[28] Nesline, F. W., and Zarchan, P., “A new look at classical vs modern homing missile guidance,” Journal of Guidance, Control, and Dynamics, Vol. 4, No. 1, 1981, pp. 78-85. doi:10.2514/3.56054.

[29] He, S., and Lee, C.-H., "Optimal Proportional-Integral Guidance with Reduced Sensitivity to Target Maneuvers," IEEE Transactions on Aerospace and Electronic Systems. doi:10.1109/TAES.2018.2824678.

[30] He, S., and Lee, C.-H., "Optimality of Error Dynamics in Missile Guidance Problems," Journal of Guidance, Control, and Dynamics, Vol. 41, No. 7, 2018, pp. 1624-1633. doi:10.2514/1.G003343.

[31] Lee, C.-H., Tahk, M.-J., and Lee, J.-I., "Generalized formulation of weighted optimal guidance laws with impact angle constraint," IEEE Transactions on Aerospace and Electronic Systems, Vol. 49, No. 2, 2013, pp. 1317-1322. doi:10.1109/TAES.2013.6494416.

[32] Weiss, M., and Shima, T., "Minimum effort pursuit/evasion guidance with specified miss distance," Journal of Guidance, Control, and Dynamics, Vol. 39, No. 5, 2016, pp. 1069-1079. doi:10.2514/1.G001623.

[33] Shima, T., Idan, M., and Golan, O. M., "Sliding-mode control for integrated missile autopilot guidance," Journal of Guidance, Control, and Dynamics, Vol. 29, No. 2, 2006, pp. 250-260. doi:10.2514/1.14951. 
[34] Weiss, M., Shima, T., Castaneda, D., and Rusnak, I., "Combined and cooperative minimum-effort guidance algorithms in an active aircraft defense scenario," Journal of Guidance, Control, and Dynamics, Vol. 40, No. 5, 2017, pp. 1241-1254. doi:10.2514/1.G002315.

[35] Zarchan, P., Tactical and strategic missile guidance, American Institute of Aeronautics and Astronautics, 2012.

[36] Lee, C.-H., Kim, T.-H., and Tahk, M.-J., "Interception angle control guidance using proportional navigation with error feedback," Journal of Guidance, Control, and Dynamics, Vol. 36, No. 5, 2013, pp. 1556-1561. doi:10.2514/1.58454.

[37] Lu, T.-T., and Shiou, S.-H., "Inverses of $2 \times 2$ block matrices," Computers \& Mathematics with Applications, Vol. 43, No. 1-2, 2002, pp. 119-129. doi:10.1016/S0898-1221(01)00278-4.

[38] Luenberger, D. G., Optimization by vector space methods, John Wiley \& Sons, 1997. 


\title{
Cranfield University
}

CERES https://dspace.lib.cranfield.ac.uk

School of Aerospace, Transport and Manufacturing (SATM)

Staff publications (SATM)

2019-01-30

\section{Minimum-effort waypoint-following guidance}

\author{
He, Shaoming
}

AIAA

He S, Lee C-H, Shin H-Y \& Tsourdos A., Minimum-effort waypoint-following guidance, Journal

of Guidance, Control, and Dynamics, Early online.

https://dspace.lib.cranfield.ac.uk/handle/1826/13904

Downloaded from Cranfield Library Services E-Repository 